\title{
NUMERICAL EXPERIMENTS ON THE $N$-BODY PROBLEM
}

\author{
S. J. AARSETH \\ Institute of Theoretical Astronomy, Cambridge, England
}

\begin{abstract}
This review first discusses the different types of numerical methods available for integrating the equations of motion of $\mathrm{N}$-body systems. It is desirable to supplement ordinary integration schemes with special treatments of close encounters using a two-body perturbation description or introducing regularizing transformations of the co-ordinates and time. Direct methods are at present limited to the study of a few hundred particles but larger systems may be investigated using Monte Carlo techniques or the Boltzmann moment equations.

$N$-body computations have been performed for a whole range of initial conditions and the general results are summarized. Numerical investigations have already clarified a number of important aspects of cluster evolution and the qualitative behaviour of small stellar systems is now quite well understood. Recent theoretical modifications have reduced the disagreement with experiments but further improvements are still needed.
\end{abstract}

\section{Introduction}

The study of $N$-body systems by numerical methods celebrates its tenth anniversary this year (von Hoerner, 1960). This relatively new development in stellar dynamics owes its existence entirely to modern technology and progress is therefore closely linked to the availability of bigger and faster computers as well as improved methods of solution. The simplicity of Newton's law of gravitation lends itself naturally to a numerical attack on the cluster problem. Given the initial distribution of individual masses, co-ordinates and velocities, the task is very well defined; e.g., to calculate in detail the behaviour of the system as a function of the time. Complete solutions to this problem can only be obtained by numerical methods based on time series expansions.

Repeated integrations of the equations of motion can readily be made by exploring a whole range of parameters for different values of the particle number. In addition, more realistic star cluster models may be simulated by allowing for stellar evolution effects and the influence of an external gravitational field. It is the aim of the direct approach to provide a better understanding of the behaviour of self-gravitating systems and use the results for dynamical interpretation of actual star cluster evolution. A considerable theoretical effort has also been directed towards the solution of this problem and the different methods of attack make it necessary to distinguish between the branches of theoretical and experimental stellar dynamics.

Although numerical studies are free from simplifying assumptions, the direct approach is limited to the simulation of small stellar systems because of heavy demands on the computing time. It is nevertheless encouraging that already the range of particle numbers has been extended to 500 which is comparable to the membership in typical galactic clusters and clusters of galaxies. The theoretical treatment becomes more reliable as the number of particles increases and a semi-empirical attack may therefore 
be made on the more formidable globular cluster problem. In addition, collisional effects are less important in rich systems which may be studied by fast numerical methods.

The problem of dynamical interpretation of the results presents considerable difficulties since it has been shown that the numerical solutions are strongly divergent (Miller, 1964; Standish, 1968). Numerical integrations of the same system at different levels of accuracy lead to an increasing separation in phase space as defined by the individual evolution. It is therefore impossible to obtain the unique solution for all time specified by a set of initial conditions. On the other hand, time reversal tests have demonstrated that reliable individual orbits can be calculated for a few crossing times, after which the numerical solutions begin to depart significantly from the original behaviour. This interval is sufficiently long to establish general features of the evolution which are consistent with results obtained over much longer times. Small systems may exhibit rather large fluctuations, however, and many cases must be studied in order to define meaningful properties. Numerical experiments can therefore be considered as repeatable under different conditions only in a statistical sense.

The evolution of richer clusters can be described with increasing confidence since each particle orbit is subject to a greater number of encounters. Thus the actual distribution of impact parameters resembles more closely the theoretical expectations which are represented by continuous expressions and more reliable comparisons can therefore be made in single cases. Cluster simulation by numerical means makes it possible to study individual interactions in considerable detail and suggest modifications of the dynamical assumptions which form the basis of theoretical treatments. The direct approach has already yielded extensive results which permit a qualitative description of the long term cluster evolution and this hopeful development should provide further stimulus for theoretical improvements.

\section{Direct Integration Methods}

A wide variety of direct methods have been used to integrate the equations of motion of the $N$-body problem

$$
\mathbf{r}_{i}=-\sum_{\substack{j=1 \\ j \neq i}}^{N} \frac{m_{j}\left(\mathbf{r}_{i}-\mathbf{r}_{j}\right)}{\left|\mathbf{r}_{i}-\mathbf{r}_{j}\right|^{3}},
$$

where the scaled mass and co-ordinates of a particle is denoted by $m_{i}$ and $\mathbf{r}_{i}$, respectively, and dots represent differentiation with respect to the time $t$. These second-order differential equations are usually written as $6 \mathrm{~N}$ equivalent equations of first order which can be solved numerically by step-wise integration. It is evident that the acceleration calculation (1) becomes very time-consuming when the particle number $N$ is large and for this reason high-order difference schemes are preferable since past information may then be used with very little additional effort. 
Writing the force per unit mass as an extrapolating polynomial of degree $n$ through $n+1$ fitting points in Newtonian form we have

$$
\mathbf{F}_{i}(t)=\sum_{\lambda=0}^{n} \mathbf{A}_{\lambda}\left(t-t_{0}\right)^{\lambda}
$$

The coefficients $A_{\lambda}$ are given by divided backwards differences weighted by constant coefficients (Wielen, 1967) and the expansion is valid over an interval $\Delta t=t-t_{0}$ which may be determined from the convergence property of Equation (2) itself. Integrating the polynomial twice we obtain the corresponding expression for the predicted position

$$
\mathbf{r}_{i}(t)=\mathbf{r}_{i}\left(t_{0}\right)+\dot{\mathbf{r}}_{i}\left(t_{0}\right)\left(t-t_{0}\right)+\sum_{m=2}^{n+2} \frac{1}{m(m-1)} \mathbf{A}_{m-2}\left(t-t_{0}\right)^{m},
$$

with the two first terms representing the position and velocity at time $t=t_{0}$. The new velocity is obtained in a similar manner.

The predicted co-ordinates and velocities may be improved by taking the difference between the $n$ 'th-order extrapolating polynomial (2) and an interpolating polynomial of order $n+1$ which is obtained after the calculation of the new force. This procedure may be referred to as a semi-iteration, since the main part of the improvement is achieved without recalculating the force based on the predicted position. In this way almost one extra order of integration is included at very little additional effort. Practical experience indicates that the gain in efficiency beyond $n=3$ or $n=4$ does not justify the use of higher orders unless extreme accuracy is demanded.

High-order schemes also require special starting procedures which are sometimes more complicated than the integration method itself. In the present formulation the derivatives may be obtained directly by explicit differentiation of Equation (1), involving relative co-ordinates and velocities only. The coefficients $A_{\lambda}$ can readily be expressed in terms of the corresponding Taylor series derivatives. This method is very convenient for starting purposes but the multiple summations required at each stage makes it rather inefficient for continuing the integration of large particle numbers (Gonzalez and Lecar, 1968).

The second essential requirement of an efficient numerical method consists of adopting a wide range of individual integration intervals. Thus the time-step appropriate to close encounters must be reduced sufficiently in order to preserve the accuracy while new positions on smooth particle orbits may be recalculated much less frequently. Suitable criteria for selecting individual steps $\Delta t_{i}$ may readily be obtained by considering the convergence of the force polynomial (2). This procedure necessitates separate prediction of all co-ordinates because of the force calculation (1) but the order used may be lower than employed by the full integration. Hence only a small proportion of the total time is used for the additional predictions but there is a considerable gain in efficiency by avoiding unnecessary force calculations. The computing time requirement is still proportional to $N^{2}$ operations per crossing time, however, and more efficient procedures should be investigated. 
The idea of individual time-steps may be carried one stage further by recalculating the contributions to Equation (1) at different times rather than simultaneously. Again the times for individual force computations may be determined from the rate of change of the corresponding contributions. For convenience all intervals are quantized into a relatively small set of categories where the steps $\Delta t_{i}$ may change by a factor of two either way. This idea has only been tried for the case $n=1$ (Hayli, 1967) and its full advantage has therefore not been realized yet.

Alternatively, distant force contributions may be calculated by the use of Legendre polynomials rather than individual summations (Aarseth, 1967). This simplification would be particularly useful for the integration of particles near the cluster centre where the net force produced by halo members is usually very small. Evidently a considerable gain in efficiency may result when the effect of distant particles is included by approximate methods, but any modifications which are introduced must be dynamically consistent with the collisional nature of the direct approach.

Numerical integrations of the equations of motion (1) cannot yield the exact solution for all time specified by a set of initial conditions. Although the error at each interval can be controlled by choosing suitable time-steps, the strong non-linearity of gravitational interactions leads to an exponential growth with a rather short time-scale (Miller, 1964; Standish, 1968).

The error amplification introduced by successive encounters may be reduced by more powerful methods to be discussed subsequently but cannot be entirely removed. Results may still be used for statistical purposes, however, provided that the evolution rate depends mainly on the overall structure and systematic errors do not enter into the calculation of individual encounters.

Practical applications make use of the ten general constants of the motion as integration tests. The conservation of the total energy, angular momentum and centre of mass motion does not by itself guarantee the accuracy of numerical solutions since errors may cancel but this is not likely to occur in general. An additional and more detailed check is provided by the time reversibility of the equations of motion. It is therefore prudent to investigate the correlation between a meaningful integration and the corresponding accuracy of the conserved quantities by time reversal tests before deciding on the final integration parameters.

\section{Special Treatments of Close Encounters}

Direct integrations of the equations of motion are very time-consuming for systems which develop high-density cores. This difficulty reflects the shortening of the central relaxation time due to the strong interaction of close neighbours. As the force fluctuations increase in strength it also becomes more difficult to conserve the integrals of motion. The choice therefore lies between terminating the calculations or making use of more powerful methods.

Detailed examinations of critical stages of evolution usually reveal the presence of close binaries or sub-groups where the members are strongly bound. The numerical 
problem becomes more serious if the binaries have long life-times since the calculation of each revolution typically takes a few hundred time-steps even if the basic elements do not change significantly over many periods. Close binaries may also suffer further changes of the semi-major axis and the replacement of the two components by the centre of mass motion would not be dynamically consistent. Instead it is natural to introduce two-body perturbation methods, replacing the dominant term by the analytical solution which is modified by the effect of the external field.

A classical variation of parameters method (Pines, 1961) has been introduced for studying close binaries in simulated clusters (Aarseth, 1970). The equation of motion for the relative co-ordinates $\mathbf{R}=\mathbf{r}_{k}-\mathbf{r}_{l}$ can be derived by writing Equation (1) for the two mass points $m_{k}, m_{l}$. Subtraction gives

$$
\ddot{\mathbf{R}}=-\frac{m_{k}+m_{l}}{R^{\mathbf{3}}} \mathbf{R}+\mathbf{F},
$$

where $\mathbf{F}$ denotes the relative perturbation acceleration.

The unperturbed solution of Equation (4) may be written in terms of the Lagrangian representation coefficients $f$ and $g$ as

$$
\mathbf{R}=f \mathbf{R}_{0}+g \dot{\mathbf{R}}_{0} .
$$

The complete motion of both components is obtained by calculating the corresponding centre of mass motion. Equation (5) and its derivative can be inverted to express the initial position and velocity vector $\mathbf{R}_{0}, \dot{\mathbf{R}}_{0}$ as functions of $\mathbf{R}$ and $\dot{\mathbf{R}}$. The relation (5) is then maintained in the presence of perturbations by integration of the variational equations

$$
\begin{aligned}
& \mathbf{R}_{0}^{\prime}=\dot{g}^{\prime} \mathbf{R}-g^{\prime} \dot{\mathbf{R}}-g \mathbf{F}, \\
& \dot{\mathbf{R}}_{0}^{\prime}=-f^{\prime} \mathbf{R}+f^{\prime} \dot{\mathbf{R}}+f \mathbf{F} .
\end{aligned}
$$

This notation uses dots to represent the two-body variation which would remain in the absence of perturbations while the perturbative variation of a quantity $h$ is denoted by $h^{\prime}$. The auxiliary variables are derived from basic elements and the perturbation effect enters through the scalar products $\mathbf{R} \cdot \mathbf{F}$ and $\dot{\mathbf{R}} \cdot \mathbf{F}$ which should remain small for the method to be efficient.

An invariant parameter

$$
\gamma=\frac{|\mathbf{F}| R^{2}}{m_{k}+m_{l}}
$$

may be introduced to indicate the relative importance of the external force field. Hence the perturbation treatment is limited to values of $\gamma$ which produce slowly varying elements and the two-body description must be replaced by direct integrations of both components if $\gamma$ exceeds a few per cent. On the other hand, it is permissible to make use of the unperturbed formulation (5) if the external effect is too small to change the relative binding energy by a significant amount. It is interesting to note that the unperturbed approximation may describe the actual motion more accurately than the 
corresponding orbit calculated by direct means because of numerical errors introduced during critical encounters. The appropriate two-body reflection in the centre of mass frame may therefore be used with advantage for the closest encounters, provided that the relative perturbation is sufficiently small.

More recently a general regularization method has been developed for the perturbed two-body problem where close encounters are treated very accurately (Kustaanheimo and Stiefel, 1965). The basic idea of regularization is to transform the equations of motion (1) into a form which removes the singularity of two-body collisions. This is achieved by first introducing generalized coordinates $\mathbf{u}$ for the relative motion $\mathbf{R}=\left(X_{1}, X_{2}, X_{3}\right)$ by the transformation

$$
\begin{aligned}
& X_{1}=u_{1}^{2}-u_{2}^{2}-u_{3}^{2}+u_{4}^{2}, \\
& X_{2}=2\left(u_{1} u_{2}-u_{3} u_{4}\right), \\
& X_{3}=2\left(u_{1} u_{3}+u_{2} u_{4}\right),
\end{aligned}
$$

which satisfies the relation

$$
R=u_{1}^{2}+u_{2}^{2}+u_{3}^{2}+u_{4}^{2} .
$$

The singularity is removed by the regularizing substitution

$$
\mathrm{d} t=R \mathrm{~d} \tau,
$$

where $\tau$ is the new fictitious time. The resulting equation of motion then becomes (Stiefel, 1967)

$$
u_{j}^{\prime \prime}=\frac{1}{2}\left(h_{0}+W\right) u_{j}+\frac{1}{4} R q_{j} \quad(j=1,2,3,4)
$$

in which $h_{0}$ is the energy constant of the two-body motion and $W$ is the work done by the external force field. The generalized perturbations $q_{j}$ are calculated from the actual components of $\mathbf{F}$ by the relations

$$
q_{j}=\sum_{i=1}^{3} \frac{\partial X_{i}}{\partial u_{j}} F_{i} .
$$

Hence the second term becomes arbitrarily small as $R \rightarrow 0$ and the solution is regular for collision orbits.

It has been demonstrated that the regularized solution is more powerful than the direct method at relatively large separations for three interacting particles (Peters, 1968). This early attempt also showed a significant improvement when regularizing the closest pair at any time for one case $N=25$ starting from rest. Only minor modifications are necessary for an efficient treatment of systems with large particle numbers.

The combined term $h_{0}+W$ in Equation (11) represents the actual binding energy per unit mass of the relative motion which reduces to

$$
h=\left[2 \sum_{j=1}^{4} u_{j}^{\prime 2}-\left(m_{k}+m_{l}\right)\right] \frac{1}{R} .
$$


Although not applicable to collision orbits this expression has the advantage of being very simple, whereas the original application involves an $N^{2}$ term which becomes prohibitive for large particle numbers.

The modified regularization treatment may be used to study the general cluster problem without experiencing the numerical difficulties due to close two-body encounters. This approach is more efficient than the perturbation method since much greater external effects can be included. Again the complete motion of both components is obtained by introducing the corresponding centre of mass. In addition, the relation (10) must be integrated in order to provide a connection between the fictitious time and the global reference time. Finally, the singularity of Equation (13) may be avoided by introducing an equation of motion for the binding energy itself, making 16 equations in all. The simultaneous use of two different methods necessitates additional programming for decision-making and reorganization but the resulting saving of time-steps and gain of accuracy is substantial (Aarseth, 1971).

The two-body regularization method is less efficient when dealing with multiple encounters since only the dominant interaction can be regularized. Although such events are less common it is nevertheless desirable to seek an improved treatment. One promising alternative consists of using the time transformation

$$
\mathrm{d} t=\frac{1}{V} \mathrm{~d} \tau
$$

for an arbitrary number of closely interacting particles giving rise to the potential $V$. Although less powerful than the full two-body regularization, the resulting equations of motion are again non-singular since Equation (14) reduces asymptotically to the form (10) in the event of a binary collision. It is also natural to include all strongly interacting particles in the transforming function $V$, leaving more distant members to be treated by the standard method (Heggie, 1971).

The recent introduction of special integration procedures is very promising for further numerical explorations of the collisional cluster problem. It is perhaps fitting that the rapid increase of available computing power should be matched by improvements in technique. Even so the task of the direct attack is formidable and alternative ideas must be considered for an extension to larger particle numbers.

\section{Simplified Methods}

In view of the severe computing time requirement for all direct methods it is desirable to explore faster alternatives which reproduce the general behaviour of $N$-body systems at particle numbers already studied. The increased speed of calculation may then be exploited to simulate the evolution of richer clusters where the adopted approximations have greater validity. Clearly the collisional approach must not sacrifice too much of the essential dynamics; e.g., encounter effects between neighbouring stars must be included. The introduction of spherical symmetry already implies a considerable simplification without loss of dynamical consistency. In addition, equal masses 
may be assumed if the more general case cannot be treated. It may be noted that neither of these assumptions would make much difference to the direct method as long as the particle description is maintained.

A fast Monte Carlo method has recently been introduced to study the evolution of spherical star clusters (Hénon, 1966). Each particle is represented by two quantities only; the binding energy and angular momentum per unit mass. Subsequent changes of the fundamental parameters are calculated by selecting a position along the orbit at random and letting the particle encounter another body chosen at random. The effect of the two-body interaction is then multiplied by an appropriate factor to give the new velocity at the end of the encounter and the next particle is treated similarly. This approach replaces the summation of $N$-1 terms in Equation (1) by the interaction of one typical member only and therefore leads to considerable saving of time. Furthermore, the time intervals $\Delta t_{i}$ which at present are equal can be a substantial fraction of the relaxation time and may easily exceed the crossing time for rich systems.

The assumption of spherical symmetry also permits a fast calculation of the potential, hence the new binding energy is readily obtained. A wide variety of steadystate initial conditions may be studied; recent results are discussed elsewhere in this volume (Hénon, 1971). It may be emphasized here that effects of multiple encounters are neglected in this procedure which can be shown to be mathematically equivalent to a solution of the corresponding Fokker-Planck equation. Direct integrations indicate that such effects are important for all cases studied so far, rut the dependence on particle number is not yet known.

A second new method for computing cluster evolution is based on the numerical solution of moment equations derived from the Boltzmann equation (Larson, 1970). Again spherical symmetry is assumed and the treatment is most suitable for equal-mass cases. Four moments are used to characterize the velocity distribution which is expanded in Legendre polynomials about a Maxwellian. Expressions for the corresponding collision terms in the Fokker-Planck equation are derived on the assumption that the velocity distribution is nearly Maxwellian. The relaxation effects in the outer parts are therefore not correctly described but encounters are also less important in regions of low density. The numerical integration of the moment equations proceeds by well tried hydrodynamical methods which give physically acceptable solutions (Larson, 1971).

Both the Monte Carlo techniques and the Boltzmann moment approach are very promising for attacking the cluster problem. The results obtained so far are encouraging and there is no doubt that such methods must be used to describe the evolution of richer systems when encounter effects are included. In the first instance, however, it is desirable to explore the region of overlap with direct methods $(N \leqslant 1000)$ in order to gain more confidence in the results.

\section{Definitions and Initial Conditions}

$N$-body integrations are most conveniently performed with scaled quantities and the results may be discussed in terms of a well-defined mean crossing time. Natural units 
are introduced by taking the gravitational constant equal to unity; in addition, the total mass and binding energy of a bound system are scaled by the relations

$$
\sum_{i=1}^{N} m_{i}=N, \quad E=-\frac{1}{4} N^{2} .
$$

This energy scaling has the advantage that results for different starting values of the virial theorem parameter may be compared at the same time once the initial evolution is over. Using the rms velocity the units (15) then define the mean crossing time

$$
t_{\mathrm{cr}}=\left(\frac{8}{N}\right)^{1 / 2}
$$

The relevant physical time in years is related to the scaled time by

$$
T \simeq 1.5 \times 10^{7}\left(\begin{array}{l}
\bar{R}^{3} \\
\bar{M}
\end{array}\right)^{1 / 2} t,
$$

where $\bar{R}$ corresponds to $r=1$ as given in pc and $\bar{M}$ is the mean particle mass in solar units.

The dynamical state of systems with small particle numbers is best described in terms of the individual binding energy and angular momentum rather than the complete distribution function $f(m, \mathbf{r}, \mathbf{r})$ used by theoretical considerations. In practice these distributions are often discussed separately; in addition, integral properties such as space density and mean velocity are studied as functions of central distance. The binding energy per unit mass is defined with respect to the inertial frame by

$$
E_{i}=-\sum_{\substack{j=1 \\ j \neq i}}^{N} \frac{m_{j}}{\left|\boldsymbol{r}_{i}-\mathbf{r}_{j}\right|}+\frac{1}{2} \dot{\mathbf{r}}_{i}^{2},
$$

using the convention $E_{i}<0$ for bound orbits. Although the binding energy is of fundamental interest in stellar dynamics, additional knowledge is often needed in order to give a better description of the overall evolution. For instance, the presence of binaries cannot be deduced directly from the energy distribution. Instead it is useful to introduce an invariant parameter $\lambda$ giving the fraction of total energy absorbed by the relative motion of $m_{k}$ and $m_{l}$ as

$$
\frac{m_{k} m_{l}}{2 a}=\frac{1}{4} \hat{\lambda} N^{2},
$$

where $a$ is the semi-major axis.

A wide variety of initial conditions have been studied for particle numbers in the range $N=3$ to $N=500$. Simple starting models can be obtained from random distributions of co-ordinates and velocities for a given mass spectrum. The velocities are then scaled to the desired value of the virial theorem parameter. This procedure does 
not introduce any special constraints on the initial configuration and simulated clusters are free to set up a dynamically consistent structure.

The restriction of starting with constant density systems is of little consequence for initial non-equilibrium configurations because of the short time-scale for significant redistribution of mass. Alternatively, more specific cases may be considered with starting conditions based on theoretical or astrophysical models. In particular it is of interest to study centrally concentrated stationary systems based on theoretical solutions of the Liouville equation (Wielen, 1967). Of the many possible mass distributions, the function $f(m) \circ m^{-2}$ is representative of the mass spectrum in young galactic clusters and is also sufficiently steep to be of general dynamical interest.

Individual particles suffer changes in the orbital parameters primarily by close encounters and the combined effect is obtained by dynamical theories for assumed distributions. Corresponding expressions may be introduced for relaxation times derived from numerical studies. Empirical definitions usually depend on the size of the sampling interval; furthermore, the contributions from two close particles may dominate unless many averages are taken. It can readily be shown that the instantaneous rate of change of the binding energy of one particle is given by

$$
E_{i}=-\sum_{\substack{j=1 \\ j \neq i}}^{N} \frac{m_{j} \dot{\mathbf{r}}_{j} \cdot\left(\mathbf{r}_{i}-\mathbf{r}_{j}\right)}{\left|\mathbf{r}^{i}-\mathbf{r}_{j}\right|^{3}}
$$

The expression (20) may be used to calculate mean relaxation times which only depend on the co-ordinates and velocities. In addition to the weighted mean defined by

$$
\dot{t}_{E}=\frac{\sum_{i=1}^{N} m_{i} \dot{\mathbf{r}}_{i}^{2}}{\sum_{i=1}^{N} m_{i}\left|\dot{E}_{i}\right|},
$$

it is instructive to study the $r$-dependence of the relaxation time since the former may be characteristic of the central region only. Repeated averages reduce spurious effects of close hyperbolic encounters but large contributions from permanent binaries would still be included for small perturbations. Instead it would be more meaningful to include the contribution from the centre of mass motion only.

Given the mean relaxation time of the whole system or a group of similar particles, the corresponding escape rate $Q$ or $Q(m)$ may be derived from the relation

$$
\Delta N=\frac{Q N \Delta t}{\tilde{t}_{E}},
$$

where $\Delta N$ denotes the number of dynamical escapers during the time interval $\Delta t$. Events leading to escape are usually well defined for isolated systems. The uncertainty in the derivation of $Q$ is therefore mainly due to statistical fluctuations if $\Delta N \ll N$. On the other hand, it is difficult to preserve uniform relaxation time definitions over the long intervals which are necessary in order to achieve significant escape rates. 


\section{General Discussion of Results}

Cluster simulations are of limited value unless it can be established that different systems exhibit a characteristic behaviour when studied over significant times. On theoretical grounds it is expected that the evolution of all cases considered by direct methods are dominated by close encounter effects, except during the early stages of non-equilibrium configurations when mass motions are important. Stellar encounters therefore provide a regulating mechanism for structural readjustments which tends to decrease initial condition differences. During this process of energy exchange some particles achieve escape velocity as the result of close encounters and are lost from isolated systems. The continuous production of escapers prevents clusters from reaching a steady state, but the evolution may nevertheless be described by a sequence of quasi-equilibrium states provided that the escape rate is small.

The overall evolution of simulated clusters proceeds in the direction of more pronounced core-halo type mass distributions. Only a small proportion of the halo members have sufficient kinetic energy to escape the system altogether, but more distant parts of the halo are gradually populated by highly eccentric orbits. The latter are mainly ejected from the central regions and the relatively long periods ensure that there is a net outward mass flux. The additional loss of fast particles from the inner region decreases the retardation on bound halo orbits and prolongs the phase of outward motion. Conditions of strict equilibrium implies an equal number of positive and negative radial velocities at all energies. Such configurations cannot be reached in practice but it is useful to study the gradual approach to equilibrium from simple initial states.

Halo orbits are approximately collisionless on the time-scale of most numerical investigations. Significant modifications of the binding energy and angular momentum therefore takes place on subsequent passages through the central region, thereby maintaining the predominance of radial motion for distant particles. The velocity anisotropy increases outwards from an approximate Maxwellian central distribution and is closely linked to the overall cluster structure. Although the transition is gradual the isotropic region can be considered completely relaxed by encounters. Consequently the halo velocity distribution may be used as a dynamical age indicator provided that distant particles were not present originally. Alternatively, it may be possible to ascribe an approximate dynamical age to the central nucleus if violent initial conditions can be ruled out.

The simulation of systems with a general mass distribution introduces many interesting features and is also more realistic. Relative evolution rates may be estimated by comparing similar equal-mass cases. Numerical half-lives of 5-50 mean crossing times have been obtained for repeated experiments with $N \leqslant 24$ (van Albada, 1968). The disruption rate is considerably increased for small clusters with moderate mass dispersions, whereas larger mass ratios do not decrease the life-times correspondingly. Encounters between unequal masses tend to promote equipartition of kinetic energy and light particles therefore have a higher probability of achieving escape velocity. 
Mass segregation takes place at the same time, however, and the longer intervals between significant encounters for light bodies counteracts the greater probability of escape in a given event.

Particles which lose kinetic energy become more strongly bound to the centre and may eventually form part of a dense nucleus dominated by heavy bodies. The strong central force field generates high velocities in order to maintain approximate equilibrium, whereas the mean velocity decreases at greater distances. This paradoxical development does not necessarily contradict the tendency towards equipartition during single encounters since the velocity of light halo bodies exceed the mean central value during passages through the nucleus. Invoking the equipartition effect, it is also possible to explain qualitatively the higher escape probability of light particles from the centre since a moderate mass ratio would be sufficient to raise the equipartition value above the local escape velocity. Further increases of the mass dispersion do not alter the character of this process; since the greater relaxation efficiency is partly compensated by a reduced number of central bodies.

The mass segregation of bound members may be estimated by comparing the mean central distance or binding energy for a given mass interval. Although differences in the mean quantities increase with time on the average, the dispersion within one group is often considerable. Thus light halo particles often become bound to the nucleus by temporary capture. Conversely, heavy bodies may be found at large central distances; this is particularly common in highly evolved systems which have expelled a significant proportion of members originally in the nucleus. The presence of heavy bodies in the outer region leads to enhanced relaxation of halo orbits but the time-scale for randomization of velocity components is usually too long to be reached by actual calculations.

Escapers carry away excess kinetic energy and therefore leave the remaining system more strongly bound. In addition, the expansion of the outer region must be compensated by a corresponding contraction of the core which itself is losing particles. Since no general configurations with more than two bodies are known to be stable it may be conjectured that the final state of evolution would tend towards one close binary with the remaining members at infinity. Evidently the time-scale for complete disruption of an isolated system may be arbitrarily large since it depends on the actual distribution of binding energies. For practical applications it is therefore more useful to estimate the half-life which is subject to less uncertainty and also within reach of machine calculations.

The final binary state conjectured above is of more than theoretical interest since the binary phenomenon appears to be fundamental to most phases of cluster evolution. At first, short lived pairs are formed at small central distances where the probability of favourable multiple encounters is relatively high. Once formed, a binary may either increase its binding or suffer disruption by further encounters. The former process dominates for heavy pairs interacting with field particles, and leads to an energy sink behaviour. A more detailed discussion is given elsewhere in this issue.

Binary activity is intimately connected with the halo expansion and escaper forma- 
tion. It has been established that most energetic escapers are associated with binary interactions (van Albada, 1968; Aarseth, 1968; Hayli, 1970). The efficiency of this mechanism favours the formation of one close central binary where the evolution measure $\lambda$ as defined by Equation (19) may eventually exceed unity (Allen, 1968; van Albada, 1968; Aarseth, 1968). Such extreme configurations have been reached after about 20-30 initial crossing times for systems containing up to 250 particles.

The addition of a galactic tidal field also speeds up the escape rate and should be included in the simulation of real star clusters. In this case many halo members which would remain bound in isolated systems escape when passing close to the Lagrangian equilibrium points (Hayli, 1970). The escape rate is increased further by the disruptive effect of passing interstellar clouds (Bouvier and Janin, 1971). Mass loss effects in heavy stars during advanced stages of physical evolution have also been considered (Wielen, 1968); more recent results of realistic cluster simulations are given elsewhere in this volume (Wielen, 1971).

\section{Comparison with Theory}

Many attempts have been made to construct theoretical cluster models which are dynamically consistent. Although the numerical experiments are restricted to rather small particle numbers, there is nevertheless some hope that a meaningful comparison can be made with theoretical predictions. As yet no theory can be said to give a completely satisfactory description of cluster evolution as understood from the numerical results. In particular it is desirable that improved treatments should include mass segregation effects as well as velocity anisotropy in the outer region. The presence of one or more close binaries also leads to increased relaxation and the production of energetic escapers not predicted theoretically.

Numerical calculations are particularly well suited for testing theoretical assumptions which are made in order to obtain complete solutions to the time dependent problem. The usual approach is to proceed from equal-mass systems to the general case by assuming the velocity distribution of different mass groups. The expected equipartition of kinetic energy does not occur for reasons discussed above. In addition, the total velocity distribution is not Maxwellian even in the simpler equal-mass case, but this approximation may still be used in the inner regions where encounters are more effective.

It is encouraging that the mean relaxation time for centrally concentrated systems is in substantial agreement with the corresponding numerical values for cases with $N=100$ (Aarseth, 1966; Wielen, 1967). A systematic dynamical comparison with the predicted dependence on particle number has not yet been attempted because of the uncertainty in defining a satisfactory numerical procedure. Instead the equivalent relaxation time due to deffections of one particle moving in a stationary field has been calculated for many constant density systems (Standish and Aksnes, 1969). The agreement with the classical Chandrasekhar theory is extremely good in the range $N=25$ to $N=2500$. This result therefore supports the assumption of relaxation by two-body 
encounters in homogeneous systems, but dynamical calculations have shown that multiple encounters are important under more realistic conditions.

Calculated relaxation times may be used to derive the mean escape rate of different mass groups as defined by Equation (22). In the absence of a standard definition of the relaxation time, however, it is more meaningful to compare the mass dependent relative escape rate per crossing time

$$
L_{m}=\frac{\Delta N_{m} t_{\mathrm{cr}}}{N_{m} \Delta t} .
$$

Although statistical fluctuations still enter through the uncertainty in the number of escapers, this effect can be minimized by repeated calculations or, alternatively, one system can be studied over longer time intervals. The two procedures are not equivalent, however, because the structure may change significantly in the latter case.

Experimental determinations of the relative escape rate have been made for several cases with $N=100$ giving $L_{m} \simeq(0.7 \pm 0.1) \times 10^{-2}$ for three mass groups (Wielen, 1967). The conclusion of nearly constant relative escape over a wide mass range is supported by one case with $N=250$ integrated over 28 mean crossing times (Aarseth, 1968); viz. $L_{m} \simeq(0.9 \pm 0.2) \times 10^{-2}$ for three groups containing $90 \%$ of the particles. The increased escape rate is mainly due to the steeper mass spectrum and the presence of a close heavy binary which dominates the evolution. Corresponding escape rates for equal-mass systems are significantly smaller even if temporary binaries are present. Thus an early calculation with two cases $N=25$ gives $L_{1} \simeq 2 \times 10^{-3}$ based on one escaper only (von Hoerner, 1963) but this value is consistent with more recent studies of small particle numbers (van Albada, 1968).

The numerical escape rates may be compared with theoretical predictions for similar systems. Analytical expressions have been derived in the case of Plummer's model for a general mass distribution with spherical symmetry and velocity isotropy (Hénon, 1969). Although mass segregation effects are not included, it is more important that the interaction between different masses has been calculated analytically.

The resulting agreement between theoretical and experimental escape rates is now better than a factor of two for the cases $N=100$ and $N=250$ discussed above. Some allowance should also be made for the effect of binaries and the higher central density in many cases, both of which would tend to decrease the discrepancy further and might in fact lead to a theoretical over-estimate. On the other hand, improved treatments of the mass segregation effect should reduce the number of light escapers in order to be consistent with the numerical escape rates. Finally, we note that the predicted escape rate remains small for equal mass systems; e.g., $L_{1} \simeq 2.6 \times 10^{-2} N^{-1}$ in good agreement with numerical results for small particle numbers.

\section{References}

Aarseth, S. J.: 1966, Monthly Notices Roy. Astron. Soc. 132, 35.

Aarseth, S. J.: 1967, Bull. Astron. 2, 47.

Aarseth, S. J.: 1968, Bull. Astron. 3, 105. 
Aarseth, S. J.: 1970, Astron. Astrophys. 9, 64.

Aarseth, S. J.: 1971, this issue, p. 118.

Albada, T. S. van: 1968, Bull Astron. Inst. Neth. 19, 479.

Allen, C.: 1968, Ph. D. Thesis, Mexico University.

Bouvier, P. and Janin, G.: 1971, in M. Lecar (ed.), Gravitational N-Body Problem, D. Reidel Publ.

Co., Dordrecht-Holland, in press.

Gonzalez, C. C. and Lecar, M.: 1968, Bull. Astron. 3, 209.

Hayli, A.: 1967, Bull. Astron. 2, 67.

Hayli, A.: 1970, Astron. Astrophys. 7, 17.

Heggie, D. C.: 1971, this issue, p. 35.

Hénon, M.: 1966, Compt. Rend. Acad. Sci. Paris 262, 666.

Hénon, M.: 1969, Astron. Astrophys. 2, 151.

Hénon, M.: 1971, this issue p. 151.

Hoerner, S. von: 1960, Z. Astrophys. 50, 184.

Hoerner, S. von: 1963, Z. Astrophys. 57, 47.

Kustaanheimo, P. and Stiefel, E.: 1965, Math. 218, 204.

Larson, R. B.: 1970, Monthly Notices Roy. Astron. Soc. 147, 323.

Larson, R. B.: 1971, in M. Lecar (ed.), Gravitational N-Body Problem, D. Reidel Publ. Co., Dordrecht-Holland, in press.

Miller, R. H.: 1964, Astrophys. J. 140, 250.

Peters, C. F.: 1968, Bull. Astron. 3, 167.

Pines, S.: 1961, Astron. J. 66, 5.

Standish, E. M.: 1968, Ph. D. Thesis, Yale University.

Standish, E. M. and Aksnes, K.: 1969, Astrophys. J. 158, 519.

Stiefel, E.: 1967, NASA Report CR-769.

Wielen, R.: 1967, Veröff. Astron. Rechen-Inst. Heidelberg, No. 19.

Wielen, R.: 1968, Bull. Astron. 3, 127.

Wielen, R.: 1971, Astrophys. Space Sci. 13, 300. 